\title{
Transient conformational remodeling of folding proteins by GroES - individually and in concert with GroEL
}

\author{
Satish Babu Moparthi • Daniel Sjölander • Laila Villebeck • \\ Bengt-Harald Jonsson • Per Hammarström • Uno Carlsson
}

Received: 10 September 2013 / Accepted: 18 September 2013 / Published online: 5 October 2013

(C) The Author(s) 2013. This article is published with open access at Springerlink.com

\begin{abstract}
The commonly accepted dogma of the bacterial GroE chaperonin system entails protein folding mediated by cycles of several ATP-dependent sequential steps where GroEL interacts with the folding client protein. In contrast, we herein report GroES-mediated dynamic remodeling (expansion and compression) of two different protein substrates during folding: the endogenous substrate MreB and carbonic anhydrase (HCAII), a well-characterized protein folding model. GroES was also found to influence GroEL binding induced unfolding and compression of the client protein underlining the synergistic activity of both chaperonins, even in the absence of ATP. This previously unidentified activity by GroES should have important implications for understanding the chaperonin mechanism and cellular stress response. Our findings necessitate a revision of the GroEL/ES mechanism.
\end{abstract}

Keywords Chaperone $\cdot$ FRET $\cdot$ Protein folding $\cdot$ Molten globule $\cdot$ MreB $\cdot$ Carbonic anhydrase

\section{Abbreviations \\ AEDANS 5-((((2-Iodoacetyl)amino)ethyl)amino) naphthalene-1-sulfonic acid}

Electronic supplementary material The online version of this article (doi:10.1007/s12154-013-0106-5) contains supplementary material, which is available to authorized users.

S. B. Moparthi · D. Sjölander · L. Villebeck · B.-H. Jonsson •

P. Hammarström $(\square) \cdot$ U. Carlsson $(\square)$

IFM-Department of Chemistry, Linköping University,

58183 Linköping, Sweden

e-mail: perha@ifm.liu.se

e-mail: ucn@ifm.liu.se

Present Address:

S. B. Moparthi

Institut Fresnel, CNRS UMR 7249, Aix-Marseille Université,

Ecole Centrale Marseille, Marseille, France
6-IAF
6-Iodoacetamidofluorescein
ANS
1-Anilino-naphthalene-8-sulfonate
FRET
$\mathrm{GuHCl}$
HCAII
Fluorescence resonance energy transfer
EPR
Guanidine $\mathrm{HCl}$
Human carbonic anhydrase II
Electron paramagnetic resonance

\section{Introduction}

In an influential study on the polypeptide flux through the GroEL/ES system, Hartl and co-workers found that it can interact with at least $30 \%$ of the cytoplasmic proteins in Escherichia coli upon heat stress [1]. Interestingly, they later identified the substrates/client proteins by using GroES fused to a histidine tag to capture the chaperonin-substrate complexes on an immobilized metal affinity chromatography (IMAC) resin [2]. However, in their approach, the IMAC resin would conceivably also capture putative GroES-substrate complexes formed between substrate and GroES alone. That possibility fueled the present study in which we characterize the interactions between protein substrates and the individual chaperonin components GroES and GroEL in all possible combinations. We have now characterized the individual interactions between the chaperonin components and the protein MreB from E. coli, an endogenous substrate previously identified as a class II substrate of GroEL/ES by the Hartl group in the aforementioned study [2]. In addition, we have chosen to use carbonic anhydrase (HCAII), as one model substrate for detailed mechanistic studies because the protein is known to interact productively with the GroEL/ES system and because its folding properties are well characterized [3-6]. Our previous papers have established an ATP-independent GroEL binding induced unfolding mechanism for avoiding misfolding of HCAII [4-6] and for $\beta$-actin by the action of TRiC [7]. Recently, similar results for these chaperonins have been reported for 
rhodanese and malate dehydrogenase (MDH) [8], supporting the generality of this mechanism.

The folding efficiency of a few proteins can be improved by the assistance of GroEL alone [9-15], whereas others require ATP to effect their release from GroEL $[16,17]$. Still others, referred to as stringent substrates, as for example ribulose-bisphosphate carboxylase/oxygenase (Rubisco), require both ATP and the co-chaperonin GroES to bring about their release [18-21].

Current knowledge suggests that non-native protein substrates interact with the apical domain of GroEL, which surrounds the opening of the central cavity. This was recently demonstrated by cryo-electron microscopy for MDH and Rubisco. The protein substrates were observed to bind multivalently to consecutive apical domains [22, 23]. GroEL primarily recognizes contiguous sequence elements or hydrophobic surfaces, such as those typically exposed in the molten globule intermediates that form in the early stages of folding due to the partial collapse of the hydrophobic residues that comprise the interior of the correctly folded molecule $[24,25]$. GroEL is a promiscuous polypeptide-binding protein which interacts with numerous proteins, both prokaryotic and eukaryotic [26]. Moreover, GroEL is capable of binding to artificial proteins of random sequence [27].

Although the GroEL/ES system has been studied in great detail, there are still fundamental discussions about its mechanism of action $[28,29]$. In this report, we have studied the interactions between two client proteins (variants of the $E$. coli MreB protein and of HCAII) and the GroEL/ES system and its individual components in various combinations and assessed their influence on the client protein conformation. In particular, we observed hitherto unreported GroES interactions with client proteins, which in retrospect logically explain reported excess of GroES concentrations during cellular stress, GroEL self-chaperoning, and restrained GroEL/ES localization at point of expression.

\section{Materials and methods}

\section{Chemicals}

5-((((2-Iodoacetyl)amino)ethyl)amino)naphthalene-1-sulfonic acid (1,5-IAEDANS) and 6-iodoacetamidofluorescein (6-IAF) were obtained from Molecular Probes, Inc., and 8-anilino-1naphthalenesulfonic acid (ANS) was purchased from Sigma. Guanidine $\mathrm{HCl}(\mathrm{GuHCl})$, a reagent grade, was obtained from MP Biochemicals. All the chemicals used were of the highest available reagent grade.

\section{Expression and purification of $\mathrm{HCAII}$ and MreB}

The HCAII single-cysteine mutants were engineered and produced as previously reported [30] using the cysteine-free
C206S pseudowild-type HCAII protein ( $\mathrm{HCAII}_{\text {pwt }}$ ) as a template. GroEL and GroES were expressed and purified as previously described $[3,5]$.

The MreB gene was cloned into the pET28a vector by restriction-free cloning [31]. Amplification was accomplished by regular PCR using a 46-base-long forward primer, which had a 24-base overlap with the vector followed by 22 bases of the $5^{\prime}$ end of the gene and a reverse primer from the $3^{\prime}$ end. Thereafter, the amplified PCR product was used as a primer pair, which anneals to the vector and extends in the linear amplification reaction. The parental plasmid was digested by DpnI and cleaves the methylated DNA. Plasmids containing the right insert and an additional His-tag were then transformed into the E. coli BL21 (DE3) cells. Then, two single and one double MreB variants that contain cysteine at selected positions were generated. Plasmids of the single variants N69C, E243C, and the double variant $\mathrm{N} 69 \mathrm{C} / \mathrm{E} 243 \mathrm{C}$ containing the correct mutation gene sequence were transformed into $E$. coli $\mathrm{BL} 21 / \mathrm{DE} 3$ cells. These cells were grown to an $\mathrm{OD}_{600}$ of $0.6-0.8$ at $37^{\circ} \mathrm{C}$ in $2 \times$ LB medium containing $60 \mathrm{mg} / \mathrm{L}$ ampicillin. Protein expression was induced by $0.5 \mathrm{mM}$ isopropyl- $\beta-d-1$-thiogalactopyranoside (IPTG), and growth was allowed to proceed at room temperature overnight. The cells were harvested by centrifugation at 4 , $400 \times g$ for $20 \mathrm{~min}$ at $4{ }^{\circ} \mathrm{C}$, and the pellet was dissolved in the lysis buffer $50 \mathrm{mM} \mathrm{NaH} \mathrm{PO}_{4}, 300 \mathrm{mM} \mathrm{NaCl}$, and $10 \mathrm{mM}$ imidazole at $\mathrm{pH} 7.5$, followed by ultrasonication $5 \times 20 \mathrm{~s}$ at $50 \mathrm{~W}$ and addition of DNAse I. The lysate was centrifuged at $14,900 \times g$ at $4{ }^{\circ} \mathrm{C}$ for $10 \mathrm{~min}$. The supernatant was applied to a Ni-NTA Fast Flow column in phosphate-buffered saline (PBS) at a flow rate of $1 \mathrm{~mL} / \mathrm{min}$. Bound his-tagged MreB was eluted with $500 \mathrm{mM}$ imidazole (in PBS buffer) at a flow rate of $1 \mathrm{~mL} /$ min, and the eluted fractions containing pure MreB variants (as determined by sodium dodecyl sulfate-polyacrylamide gel electrophoresis (SDS-PAGE) analysis) were dialyzed against $1 \mathrm{mM}$ $\mathrm{NaH}_{2} \mathrm{PO}_{4}, 10 \mathrm{mM} \mathrm{NaCl}$, and $0.1 \mathrm{mM}$ dithiothreitol (DTT) phosphate buffer at $4{ }^{\circ} \mathrm{C}$ in 6-h intervals for $24 \mathrm{~h}$.

\section{Reactivation experiments}

Reactivation of denatured HCAII was measured by the colorimetric $\mathrm{CO}_{2}$ hydration assay. HCAII $(9.35 \mu \mathrm{M})$ was denatured for $2 \mathrm{~h}$ in $3.3 \mathrm{M} \mathrm{GuHCl}\left(0.1 \mathrm{M}\right.$ Tris- $\left.\mathrm{H}_{2} \mathrm{SO}_{4}, \mathrm{pH} 7.5\right)$. Reactivation was started by dilution of the denatured protein solution to $0.3 \mathrm{M} \mathrm{GuHCl}$ and the protein to $0.85 \mu \mathrm{M}$ by rapid addition of the dilution buffer containing $0.1 \mathrm{M}$ Tris- $\mathrm{H}_{2} \mathrm{SO}_{4}$ (pH 7.5), $10 \mathrm{mM} \mathrm{KCl}$, and $10 \mathrm{mM} \mathrm{MgSO}_{4}$ and when indicated the same concentrations of GroEL, GroES, and GroEL/ES/ ATP as in the steady-state fluorescence measurements.

\section{Labeling of HCAII Cys mutants with IAEDANS}

Labeling followed by affinity chromatography purification was performed as reported by Svensson et al. [30]. All 
mutants were labeled in the unfolded state, and the labeling was quenched after $24 \mathrm{~h}$ incubation with shaking, by the addition of $10 \mathrm{mM} \mathrm{2-mercaptoethanol,} \mathrm{followed} \mathrm{by} \mathrm{refolding.}$ The obtained degree of labeling was $90-100 \%$, based on absorption measurements of HCA II at $280 \mathrm{~nm}$ and of AEDANS at $337 \mathrm{~nm}$ using the molar extinction coefficients $54,800 \mathrm{M}^{-1} \mathrm{~cm}^{-1}$ for HCAII and $6,100 \mathrm{M}^{-1} \mathrm{~cm}^{-1}$ for AEDANS.

\section{Labeling of MreB Cys mutants with fluorescein}

The labeling of MreB by 6-IAF was performed in the native state by incubating purified MreB in 0.1 M PIPES (pH 6.9) with a 10-fold excess of 6-IAF for $2 \mathrm{~h}$. Removal of excess 6IAF was performed by gel filtration (PD10, G25 Sephadex column, GE-Healthcare) and two rounds of dialysis. The degree of labeling was calculated by determination of free thiol groups by Ellman's reagent using an extinction coefficient of $14,150 \mathrm{M}^{-1} \mathrm{~cm}^{-1}$ at $412 \mathrm{~nm}$ [32] and was determined to be $>90 \%$ for all variants.

\section{Steady-state fluorescence measurements of HCAII}

$\mathrm{GuHCl}$ unfolding fluorescence measurements were performed with $0.85 \mu \mathrm{M}$ solutions of labeled HCAII incubated overnight in varying concentrations of $\mathrm{GuHCl}$ buffered with $0.1 \mathrm{M}$ Tris- $\mathrm{H}_{2} \mathrm{SO}_{4}, \mathrm{pH}$ 7.5. Fluorescence spectra were recorded using a Hitachi F-4500 spectrofluorometer, exciting at $295 \mathrm{~nm}$ and monitoring emission at 310-570 $\mathrm{nm}$. Excitation was also done at $350 \mathrm{~nm}$ with recording of the AEDANS emission in the range $380-570 \mathrm{~nm}$. Slits for both emission and excitation light were set at $5 \mathrm{~nm}$. The FRET intensity ratio $\left(I_{295} / I_{350}\right)$ was calculated by dividing the AEDANS emission maximum intensity following excitation at $295 \mathrm{~nm}$ (sensitized FRET) by the AEDANS emission maximum intensity following excitation at $350 \mathrm{~nm}$ [5].

To study the steady-state refolding kinetics of AEDANSlabeled HCAII mutants, the protein was first unfolded in $3.3 \mathrm{M} \mathrm{GuHCl}\left(0.1 \mathrm{M}\right.$ Tris- $\left.\mathrm{H}_{2} \mathrm{SO}_{4}, \mathrm{pH} 7.5\right)$ for $2 \mathrm{~h}$ with a protein concentration of $9.35 \mu \mathrm{M}$. The spontaneous refolding reaction was initiated by dilution of the denaturant to a final concentration of $0.3 \mathrm{M} \mathrm{GuHCl}\left(0.1 \mathrm{M}\right.$ Tris- $\mathrm{H}_{2} \mathrm{SO}_{4}, \mathrm{pH}$ 7.5; protein concentration $0.85 \mu \mathrm{M}$ ) directly in an observation cuvette, followed by manual mixing, and the spectral scan was finished in $60 \mathrm{~s}$ at $21{ }^{\circ} \mathrm{C}$. After the completion of the refolding process $(3 \mathrm{~h})$, the fluorescence spectrum was registered again. The same procedure was followed in chaperoninassisted refolding studies, except that GroEL and GroES were included in the dilution buffer $\left(0.1 \mathrm{M}\right.$ Tris- $\left.\mathrm{H}_{2} \mathrm{SO}_{4}, \mathrm{pH} 7.5\right)$ : GroEL in a 1.1-fold and GroES in 2.2-fold molar excess over the HCAII protein substrate. The same chaperonin concentrations were maintained in the refolding experiments assisted by GroEL + GroES and GroEL/ES/ATP, where $1 \mathrm{mM} \mathrm{Mg-ATP}$ (freshly dissolved) was used. All experiments were repeated two or three times, and the standard deviations of the average values were calculated.

Effects on compactness of refolding HCAII by Trp-AEDANS FRET measurements

Labeling the protein with AEDANS resulted in pronounced FRET as evident through decreased quantum yield of the Trp donors (Supplementary: Fig. S1a) which is especially notable in the native $(0.3 \mathrm{M} \mathrm{GuHCl})$ sample and less pronounced in the unfolded state $(3.3 \mathrm{M} \mathrm{GuHCl})$. Comparing the fluorescence intensity of the acceptor AEDANS emission maximum (around $500 \mathrm{~nm}$ ) after sensitized excitation of the Trp residues $(295 \mathrm{~nm})$ or the AEDANS probe $(350 \mathrm{~nm})$, it is evident that there is a pronounced FRET between the Trp-AEDANS fluorophores (Supplementary: Fig. S1a). The ratio of these fluorescence intensities, i.e., the sensitized AEDANS fluorescence excited at these wavelengths $\left(I_{295} / I_{350}\right)$, can, as in a previous study [5], be used as a measure of FRET. This value is found to be high in the folded protein (at $0.3 \mathrm{M} \mathrm{GuHCl}$ ) and low in the unfolded protein variants (at $3.3 \mathrm{M} \mathrm{GuHCl}$ ). Thus, the efficiency of FRET depends upon the location of the Trp donors and AEDANS acceptors in the protein. $\mathrm{GuHCl}$ equilibrium unfolding curves, as monitored by FRET, are shown in Supplementary: Fig. S1b for the various AEDANS-labeled HCAII variants. These curves follow the corresponding unfolding transition curves of HCAII obtained by other parameters (CD ellipticity, intrinsic Trp fluorescence intensity and wavelength shift, enzyme activity, UV absorbance) [33-36], demonstrating that this FRET parameter is a reliable measure of the degree of unfolding. Thus, these curves can be used as references when following changes in compactness during interaction with GroEL, GroES, GroEL+GroES, and GroEL/ES/ATP. The FRET values obtained at $0.3 \mathrm{M} \mathrm{GuHCl}$ and $3.3 \mathrm{M} \mathrm{GuHCl}$ are assumed to correspond to the native state (fraction folded $=1$ ) and the fully unfolded state of HCAII (fraction folded=0), respectively, since the folding curves show merely minor changes beyond these $\mathrm{GuHCl}$ concentrations.

Fluorescence anisotropy and homo-FRET of MreB mutants

Fluorescence measurements were performed for GroEL or GroES containing samples $(600 \mu \mathrm{L})$ with $0.4 \mu \mathrm{M}$ MreB, $1.6 \mu \mathrm{M}$ GroEL, $2.8 \mu \mathrm{M}$ GroES, and $160 \mu \mathrm{M}$ ATP in $0.1 \mathrm{M}$ Tris- $\mathrm{HCl}, 10 \mathrm{mM} \mathrm{KCl}, 10 \mathrm{mM} \mathrm{MgCl}_{2}$, pH 7.5. The samples were prepared as follows: After addition of $1 \mathrm{mM}$ DTT to GroEL or GroES used at different conditions, the sample was incubated at $30{ }^{\circ} \mathrm{C}$ for $15 \mathrm{~min}$. 6-IAF-labeled and denatured MreB (in $4 \mathrm{M} \mathrm{GuHCl}$ ) was mixed with unlabeled, denatured MreB in 1:4 molar ratios and was subsequently diluted 125 300-fold into the GroEL/ES-containing samples. The 
refolding reaction was allowed to proceed for $45 \min$ at $30{ }^{\circ} \mathrm{C}$. Soluble aggregates were removed by centrifugation at 17 , $700 \times g$ for $5 \mathrm{~min}$. In the case of GroEL/ES/ATP- mediated MreB refolding, ATP was added to GroEL/ES/MreB sample, incubated at $30{ }^{\circ} \mathrm{C}$ for $15 \mathrm{~min}$, and centrifuged as described above before the spectra were recorded.

The steady-state fluorescence anisotropy spectra were recorded on a Hitachi F-4500 spectrofluorometer at $21{ }^{\circ} \mathrm{C}$, with slits of 5 and $10 \mathrm{~nm}$ for emission and excitation, respectively. The emission wavelength was set to $524 \mathrm{~nm}$, and the excitation spectra were collected in the region $460-510 \mathrm{~nm}$ at the four different polarization configurations, $\mathrm{VV}, \mathrm{VH}, \mathrm{HH}$, and HV. The distances between the inserted probes were calculated by use of steady-state anisotropy and homo-FRET as previously described in detail [37].

Fluorescence measurements of AEDANS in an ethanol/water gradient

Ethanol (100\%) was diluted with milli-Q water in increments of 10 volume percentages down to pure water and subsequently purged by $\mathrm{N}_{2} .1,5$-IAEDANS $(0.4 \mu \mathrm{M})$ with a 3 -fold molar excess of reduced glutathione was added to each ethanol/ water solution. Prior to fluorescence measurement, the ethanol/water solutions were extensively purged by $\mathrm{N}_{2}$ gas in the cuvette to remove $\mathrm{O}_{2}$ that quenches the fluorescence. Fluorescence emission was recorded on a Hitachi-4500 spectrofluorometer at $21{ }^{\circ} \mathrm{C}$ using slits of $10 \mathrm{~nm}$ for excitation and $5 \mathrm{~nm}$ for emission. Excitation wavelength was set to $350 \mathrm{~nm}$, and the emission was recorded between 400 and $650 \mathrm{~nm}$. A color gradient of the fluorescence light was created in the free vector software InkScape 0.47 , using the gradient tool and selecting blue and red as the two extreme colors. The hexadecimal color value in each rectangle (wavelength) was determined by averaging the colors in a circular area of $15 \mathrm{pts}$ at $200 \%$ magnification. Values for the dielectric constants of the corresponding ethanol/water solutions used in the resulting image and graph were obtained from Landolt-Börnstein [38]. The hexadecimal values obtained in InkScape was then used in the free molecular viewing software YASARA 9.11.23 (http://www.yasara.org) to color the amino acid residues of HCAII (PDB Code: 2cba [39] that had been labeled with AEDANS; the final image was rendered in POV-Ray 3.6 (http://www.povray.org).

\section{Stopped-flow FRET measurements of HCAII}

FRET measurements were performed using the seven Trp residues in HCA II as donors and AEDANS fluorophores as acceptors (multidonors-single acceptor approach) inserted by site-directed Cys labeling at various specific positions [5]. Since the Trp residues are distributed evenly throughout the protein (11) (in positions 5, 16, 97, 123, 192, 209, and 245) and the
AEDANS probes are inserted at different positions in the protein structure in a variety of microenvironments $(\mathrm{H} 10 \mathrm{C}$ in the N-terminal, H64C, N67C, L79C in the center of the protein, and $\mathrm{N} 253 \mathrm{C}$ in the $\mathrm{C}$-terminal end), FRET values will reflect the compactness of the overall HCAII molecule but could also give localized information about conformational changes accompanying the folding process. Due to the lack of Trp residues in GroEL and GroES, it is possible to uniquely determine the TrpAEDANS FRET in HCAII in the presence of GroEL/ES. The stopped-flow experiments to monitor the tryptophan-AEDANS fluorescence were performed at $21{ }^{\circ} \mathrm{C}$ on an SX.18MV-R stopped-flow reaction analyzer (Applied Photophysics) in a $20-\mu \mathrm{L}$ cell. The wavelengths 295 and $350 \mathrm{~nm}$ with a bandwidth of $5 \mathrm{~nm}$ were used for Trp and AEDANS excitation, respectively. Emission was collected via the fluorometric port of the cell, and an optical filter was used at the emission window to monitor all fluorescence at wavelengths higher than $445 \mathrm{~nm}$, i.e., AEDANS fluorescence excited through Trp FRET $(295 \mathrm{~nm})$ or directly $(350 \mathrm{~nm})$. Before the measurements, the photomultiplier tube voltage and offset were adjusted using the folded protein $(0.85 \mu \mathrm{M})$ in $0.3 \mathrm{M} \mathrm{GuHCl}\left(0.1 \mathrm{M}\right.$ Tris- $\mathrm{H}_{2} \mathrm{SO}_{4}$, $\mathrm{pH} 7.5$ ) to bring the signal to a baseline with the intensity zero in the window -4 to 4 . The protein $(9.35 \mu \mathrm{M})$ was denatured for $2 \mathrm{~h}$ by $3.3 \mathrm{M} \mathrm{GuHCl}\left(0.1 \mathrm{M}\right.$ Tris- $\left.\mathrm{H}_{2} \mathrm{SO}_{4}, \mathrm{pH} 7.5\right)$ at $21^{\circ} \mathrm{C}$. Refolding was initiated by dilution to $0.3 \mathrm{M} \mathrm{GuHCl}$ and a protein concentration of $0.85 \mu \mathrm{M}$ by a $1: 10$ dilution with $0.1 \mathrm{M}$ Tris- $\mathrm{H}_{2} \mathrm{SO}_{4}$ buffer, $\mathrm{pH}$ 7.5. The same concentrations of AEDANS-labeled HCAII variants, GroEL, GroES, GroEL+ GroES, and GroEL/ES/ATP, as in the steady-state fluorescence measurements were used. Data were recorded by monitoring the fluorescence signal in real time from the end of the stoppedflow push, and the first data point was collected at $50 \mathrm{~ms}$. Seven consecutive runs were made for each sample of the protein variants, of which the first two were discarded because these often showed signs of being influenced by the diffusion in the tubing. Moreover, as a minimum two such experiment series were performed from which standard deviations were calculated. All data were collected with the drive pressure held constant through the whole measurement because this gave more reproducible data and did not influence the refolding. The pulse sequence was used in the experiment enabling a baseline to be recorded with no protein in the cell (for clarity the baselines are not shown in the figure).

\section{Stopped-flow ANS fluorescence measurements of HCAII}

The time course of ANS binding to the protein upon adsorption was registered by stopped-flow fluorescence measurements with the same stopped-flow instrument setup as described above. Excitation was performed at $360 \mathrm{~nm}$ and emission was collected via the fluorometric port of the cell through an optical filter with a cutoff at $455 \mathrm{~nm}$. Before the measurements, the photomultiplier tube voltage and offset 
were adjusted using the folded protein $(0.85 \mu \mathrm{M})$ and a 10 fold molar excess of ANS over HCAII in $0.3 \mathrm{M} \mathrm{GuHCl}$ (0.1 M Tris- $\left.\mathrm{H}_{2} \mathrm{SO}_{4}, \mathrm{pH} 7.5\right)$ to bring the signal to a baseline with the intensity zero in the window -4 to 4 . Refolding with and without the chaperonin components in the presence of ANS was carried out as described above for the stopped-flow AEDANS-HCAII variants except that unlabeled HCAII was used as the protein substrate. To study the formation of the molten globule in the early stages of the refolding process, the refolding process was initiated by mixing with the dilution buffer containing ANS in a 10-fold molar excess over protein $(0.85 \mu \mathrm{M})$. The binding of ANS to the refolding protein was monitored for $50 \mathrm{~s}$, and the data reported for the kinetics of the interaction between the folding protein and ANS originate from the first collected data point $(50 \mathrm{~ms})$.

Expression, purification, and labeling of GroES-98C

Expression and purification of GroES-98C mutant was performed using the GroES-98C expression plasmid from Dr. George H. Lorimer, transformed into BL21 E. coli. The protein was expressed using $200 \mathrm{mM}$ IPTG induction at $30{ }^{\circ} \mathrm{C}$. The cell lysate was heated to $75^{\circ} \mathrm{C}$ for $10 \mathrm{~min}$, followed by cooling to room temperature and centrifugation. The supernatant $\mathrm{pH}$ was adjusted to 4.5 with Tris-acetate buffer and applied on a DEAE-Sepharose column and eluted with a $\mathrm{NaCl}$ gradient. Fractions containing GroES protein was selected from pure GroES bands on SDS-PAGE.

Fractions were thereafter selected showing minor absorbance at $280-260 \mathrm{~nm}$ retaining strong labeling with the Bradford protein assay. The protein was dialyzed versus $10 \mathrm{mM}$ Tris$\mathrm{HCl} \mathrm{pH} 7.4$ buffer. One round of DTT $(1 \mathrm{mM})$ and $0.5 \mathrm{M} \mathrm{GuHCl}$ "scrubbing" of putatively bound substrates and C98 modifiers was performed followed by PD-10 purification in PBS pH 7.4. The newly scrubbed GroES-98C protein was labeled in the native state using a 10-fold molar excess 1,5-IAEDANS for $1 \mathrm{~h}$, and the protein was purified using a PD-10 column. Labeling efficiency was determined using both MALDI-TOF MS and Bradford protein assay and $\varepsilon=5,700 \mathrm{M}^{-1} \mathrm{~cm}^{-1}$ at $337 \mathrm{~nm}$ for IAEDANS. The labeling efficiency was determined to be 5.9 AEDANS molecules per GroES heptamer.

\section{FRET refolding assay by Trp-AEDANS FRET (GroES-98C-AEDANS)}

Refolding of unlabeled HCA II (H64C/C206S) was performed by initial unfolding of HCA II in $3.3 \mathrm{M} \mathrm{GuHCl}$ for $>2 \mathrm{~h}$. Refolding was initiated through rapid dilution in PBS buffer $\mathrm{pH} 7.4$ in the presence or absence of $1.0 \mu \mathrm{M}$ GroES98C-AEDANS.

Titration assay Stepwise additions were made in 5 min intervals by manual mixing by adding $5.5 \mu \mathrm{L}$ of unfolded HCA II (from a $9.35 \mu \mathrm{M}$ stock) to a $600-\mu \mathrm{L}$ solution with and without GroES-C98-AEDANS, followed by direct measurement of Trp fluorescence. Excitation was performed at $295 \mathrm{~nm}$ using $5 / 5 \mathrm{~nm}$ slits and $700 \mathrm{~V}$ PMT; emission was recorded between $310-570 \mathrm{~nm}$. Each scan was finished within $60 \mathrm{~s}$ after addition of HCAII.

Trp-AEDANS FRET of "other clients" Refolding of unlabeled proteins (bovine serum albumin (BSA), bovine $\gamma$-globulin, chicken lysozyme, bovine DNAse I) was performed by initial unfolding in $5.0 \mathrm{M} \mathrm{GuHCl}$ for $48 \mathrm{~h}$. Refolding was initiated through rapid dilution to a final concentration of $0.5 \mu \mathrm{M}$ protein in PBS buffer $\mathrm{pH} 7.4$ (with residual $\mathrm{GuHCl}$ concentrations of $0.03-0.1 \mathrm{M}$ ) in the presence or absence of $1.0 \mu \mathrm{M}$ GroES-98C-AEDANS. Excitation was performed at $295 \mathrm{~nm}$ using 5/5 nm slits and $700 \mathrm{~V}$ PMT; emission was recorded between 310 and $570 \mathrm{~nm}$.

\section{Results}

Interactions between the components of the GroEL/GroES system and the protein substrate MreB from E. coli We unexpectedly found that GroES alone was able to bind and interact with the protein substrate in our earlier studies of the mechanism of action of the GroEL/ES system using the protein folding model protein HCAII as a client protein (see data below) [40].

Therefore, to explore if interactions with GroES alone may be important in vivo, we chose an endogenous client protein in E. coli cells that has been identified by Hartl and coworkers as a class II substrate for GroEL/ES [2]. We herein chose the cytoskeletal MreB as a test case. The interactions of the MreB homolog $\beta$-actin with the eukaryotic chaperonin TriC and the GroE system have been carefully characterized by us [7]. In the latter study, the interactions between $\beta$-actin and TRiC were successfully characterized by measurements of the changes in fluorescence anisotropy and in FRET from the fluorescein-labeled variants of actin upon their binding to the chaperonin. For that reason, we introduced cysteines at two positions in a cysteine-free variant of MreB leading to the three variants, N69C, E243C, and N69C/E243C, in which the cysteines were subsequently modified by a fluorescein fluorophore (Fig. 1a). The anisotropy for all MreB variants is higher upon refolding in the presence of GroES than upon spontaneous refolding (Fig. 1b) showing the formation of a complex which tumbles more slowly than MreB itself, i.e., MreB and the 70-kDa GroES form a GroES/MreB complex. The large increase in anisotropy at the other experimental conditions shows that MreB forms a very slowly tumbling GroEL/MreB complex with the $800-\mathrm{kDa}$ GroEL alone and a ternary GroEL/ES/MreB complex in the presence of both 
Fig. 1 Steady-state averages of the anisotropy and distance of MreB variants after refolding. a Molecular model representing $E$. coli MreB showing sites, N69C and E243C, chosen for sitespecific fluorescein labeling. The corresponding positions were selected by sequence alignment in which $E$. coli MreB was modeled on top of the crystal structure of Thermatoga maritima MreB (1JCE.pdb [62]) in YASARA v9.11.23 (www.yasara.org). b Anisotropy averages of the fluorescein-labeled MreB single variants $\mathrm{N} 69 \mathrm{C}, \mathrm{E} 243 \mathrm{C}$ and double variant N69C/E243C during various chaperonin conditions. The GroE components were present from the beginning before addition of the denatured MreB during refolding: GroEL alone (green), GroES alone (white), GroEL+ GroES (blue), GroEL/ES/ATP (red), and in comparison with the spontaneous refolding (black). c Steady-state FRET distances (in ångström) determined by homoFRET measurements. The same color code as in $\mathbf{b}$ a

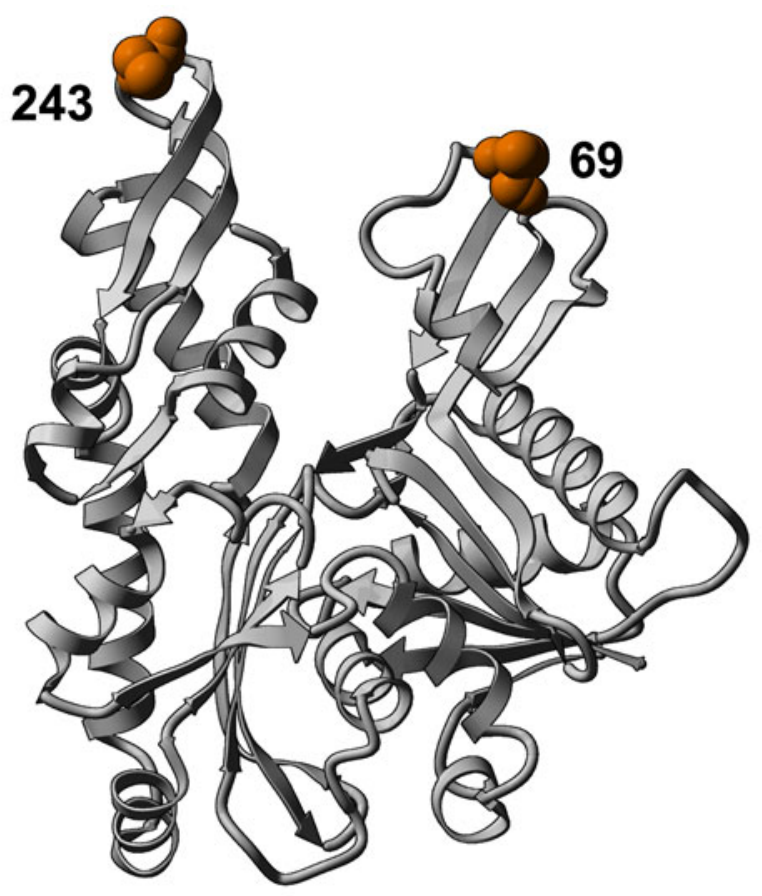

b

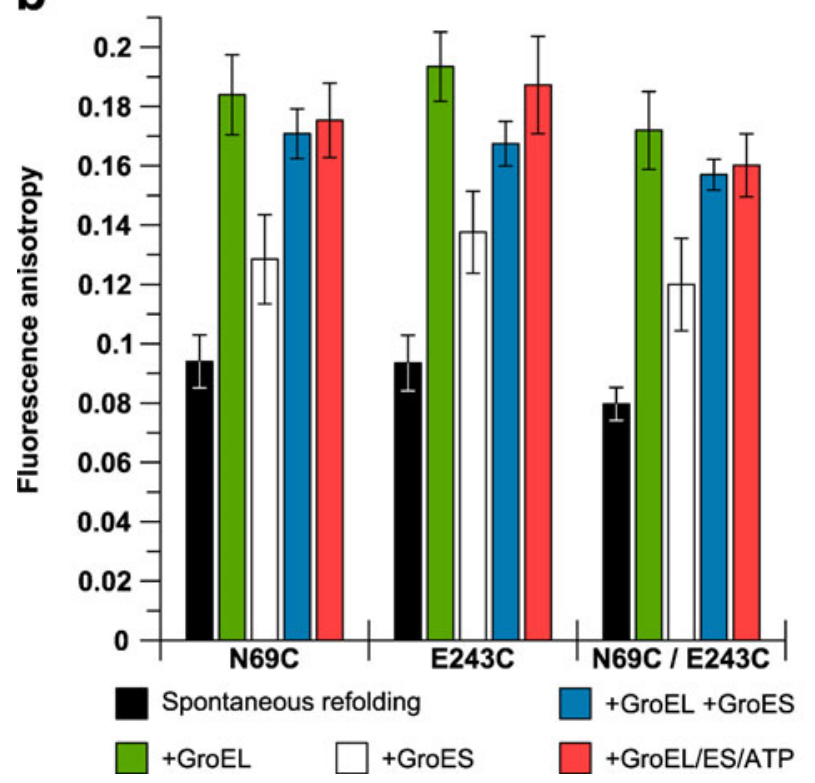

C

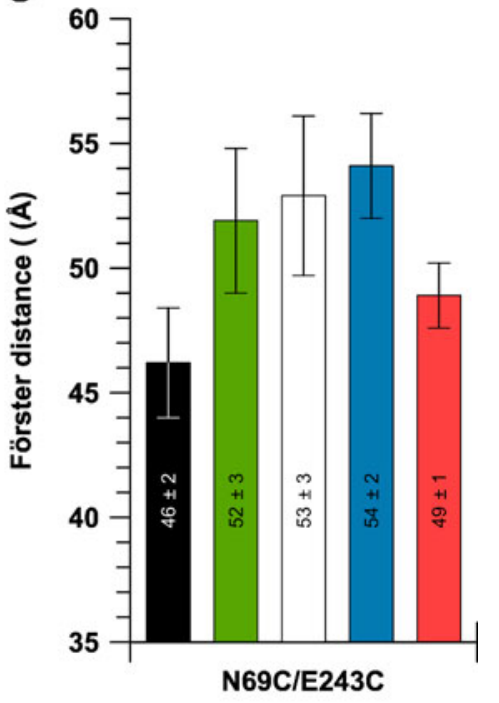

chaperonin components, and after addition of ATP, a GroEL/ ES/MreB/ATP complex is observed (Fig. 1b). Thus, these results unequivocally show that GroES alone can form complexes with natural substrates to the chaperonin, in agreement with our conclusions from the studies with HCAII as a substrate (for details, see below). Interestingly, the differences between the observed anisotropies show that distinct GroES/MreB, GroEL/MreB, and GroES/EL/MreB complexes are formed (most notable for the E243C-variant in Fig. 1b), which clearly indicates a client protein induced formation of a ternary GroES/EL/MreB complex with no requirement for ATP. The fluorescence anisotropy from the doubly labeled variants is affected by a distance-dependent homo-FRET between the two fluorophores [7]. A calculation of the distances between the fluorophores in the N69C/E243C variant under the different conditions is presented in Fig. 1c. The most apparent results from a comparison of those distances are that the distance increases upon formation of all the GroES/ MreB, GroEL/MreB, and GroES/EL/MreB complexes when compared to the spontaneous refolding reaction and notably the addition of ATP to the GroES/EL/MreB complex induces a compaction of the MreB substrate (i.e., the distance between the 
fluorophores decreases from 54 to $49 \AA$ ) well in accordance with our data on the TriC-mediated $\beta$-actin folding process [7].

Both GroES and GroEL interact with the kinetic molten globule of HCAII Previously, we have established that GroEL forms a 1:1 complex with the molten globule state of HCAII under equilibrium conditions (at $50^{\circ} \mathrm{C}$ ) and that this interaction leads to an expansion of the structure of both substrate and GroEL [4-6, 41]. Additional evidence supporting a binding induced unfolding model for GroEL was recently obtained using a folding incompetent variant of alanine glyoxylate aminotransferase [42]. There is, however, no data available on how the individual chaperonin components GroEL and GroES affect the conformation of HCAII during the folding process. Since the folding reaction of HCAII is very well characterized both at equilibrium and kinetically, this protein would serve as an excellent model substrate in a detailed study of the stepwise interactions between the various GroE components and the protein substrate [43]. Therefore, we initiated a kinetic study to investigate how the kinetic molten globule intermediate was affected by interactions with each of GroEL and GroES. Previously, it has been demonstrated that the formation of the molten globule intermediate during folding of HCAII and bovine CAII can be probed by the hydrophobic fluorescent probe ANS [44-46]. Thus, the effects on refolding of HCAII by the presence of the different chaperonin components were monitored by registering ANS fluorescence after initiation of refolding by stopped-flow mixing (Fig. 2). The presence of GroEL led to a loss of ANS binding compared to the spontaneous refolding process, showing that the HCAII substrate interacts with GroEL and that this interaction shields hydrophobic patches on HCAII from interaction with ANS. Thus, formation or exposure of an aggregation-prone molten globule intermediate appears to be reduced by interaction with GroEL. Refolding in the presence of GroES also led to significantly less binding of ANS verifying that GroES alone forms a complex with the protein substrate HCAII at an early stage of refolding. Interestingly, the effect by GroEL and GroES on ANS binding to HCAII is partly additive because the binding to ANS in the simultaneous presence of GroEL and GroES is less than with each of the individual components (Fig. 2). Thus, it seems as if some of the interactions with GroES are maintained when HCAII is bound in a ternary complex composed of GroEL/ES/HCAII, i.e., both chaperonin components contribute to a reduction of the exposed hydrophobic patches typical for the molten globule.

How do GroEL and GroES influence one-another's binding to the client protein? Local environmental changes in the client protein arising from interaction with the chaperoning components can be monitored by use of the fluorescent probe AEDANS introduced at various positions in the structure. The fluorescence emission maximum (Stokes shift) of

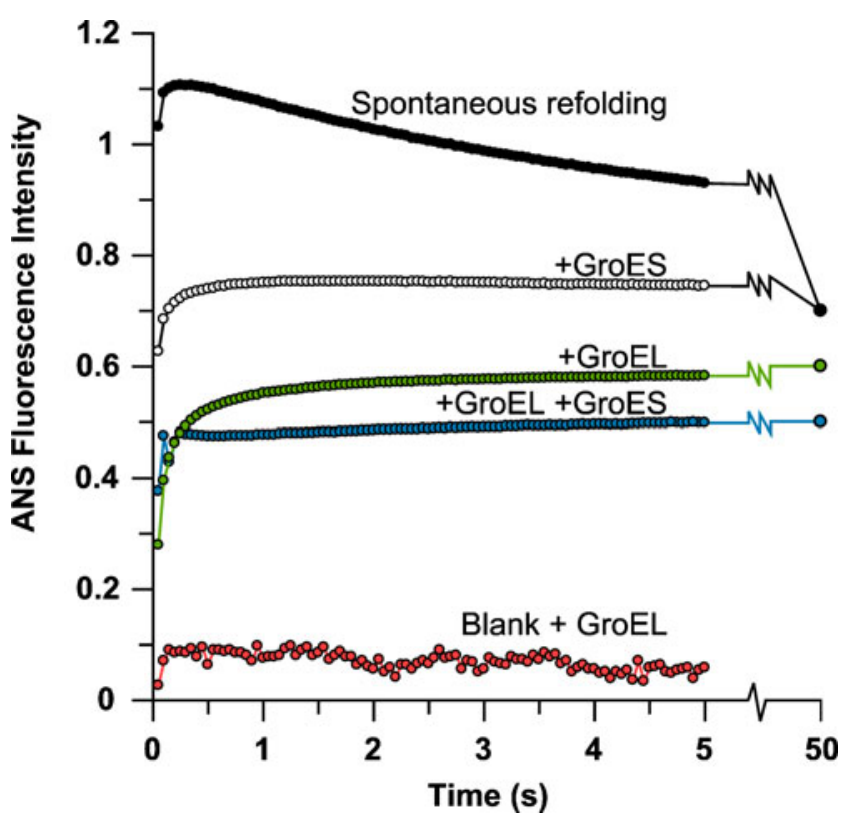

Fig. 2 Effect on the HCAII molten globule intermediate during refolding. Stopped flow kinetic traces are shown for ANS fluorescence upon binding to the protein substrate during refolding in the presence and absence of various chaperonin components

AEDANS is highly sensitive toward the local polarity of the dye's environment as illustrated in Fig. 3a. In aqueous solution, it has an emission maximum at $505 \mathrm{~nm}$, whereas in the interior of proteins, it typically exhibits a blue shift toward $460 \mathrm{~nm}$ [47]. Therefore, AEDANS was introduced at five engineered cysteine positions spanning the HCAII molecule at positions 10,64, 67, 79, and 253. The environmental changes at these positions upon interaction with the chaperon components are summarized in Fig. 3b. The emission wavelength maxima of AEDANS within the native protein showed a wide distribution (483-497 nm), as expected from the differing polarities in the selected microenvironments in the HCAII molecule corresponding to dielectric constants of 47-72 (Fig. 3a). In the unfolded state, the AEDANS fluorescence indicates a highly polar environment $(500-505 \mathrm{~nm})$ for all positions (Fig. 3b), showing dielectric constants of 72-80, near water (Fig. 3a). A comparison of the results emanating from interactions in the presence of GroEL, GroES, GroEL+ GroES, and upon spontaneous refolding (after $1 \mathrm{~min}$ of refolding) shows that the pattern is different for all these situations (Fig. 3b, c), i.e., the microenvironments at virtually all individual positions differ substantially in all pairwise comparisons between these situations. First, the comparison shows that interaction with GroEL and GroES alone leads to differences at all positions. Second, in the presence of both GroEL and GroES, the pattern changes completely because no microenvironment is similar to any of the corresponding microenvironments observed in the GroEL/HCAII and GroES/HCAII complexes. Notably the microenvironment of residues 10-79 becomes very similar (and nonpolar) in the 
b

a

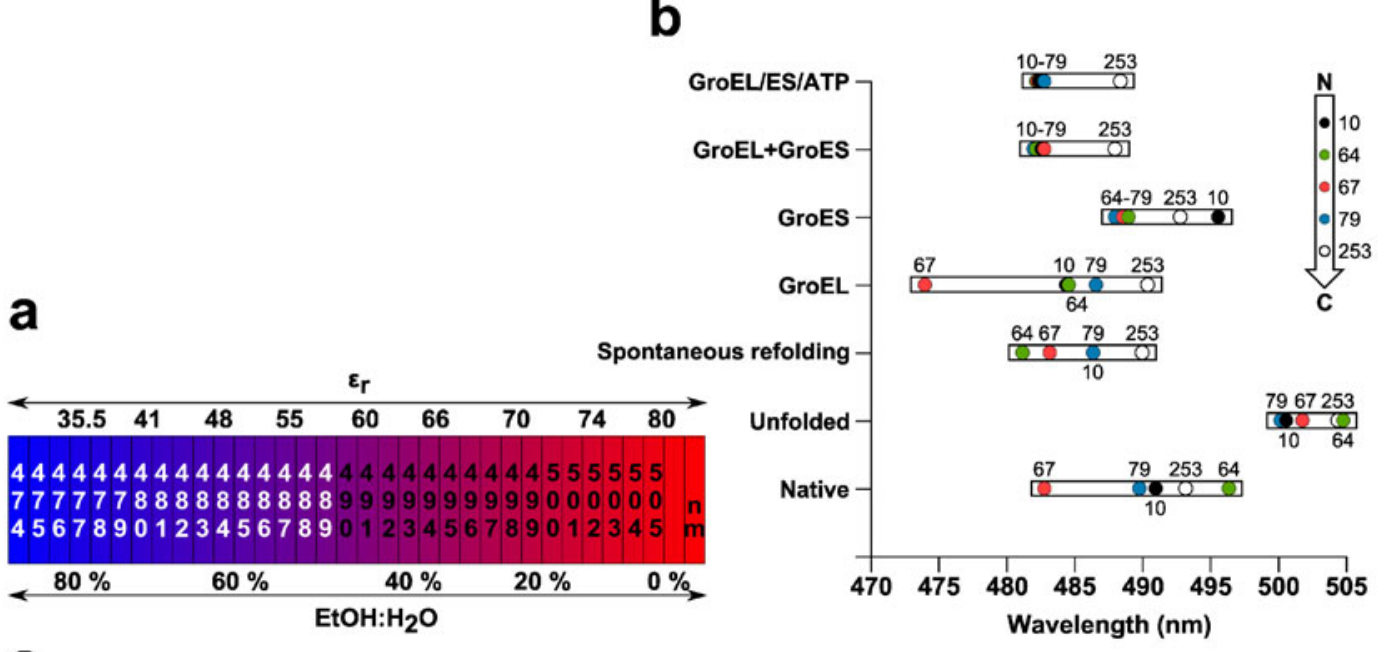

C

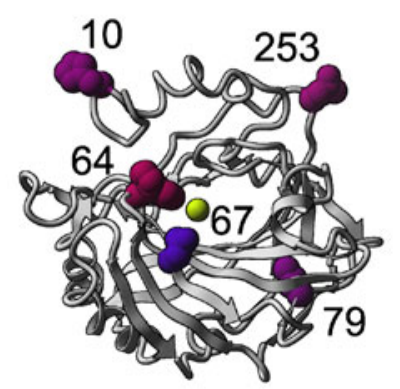

Native

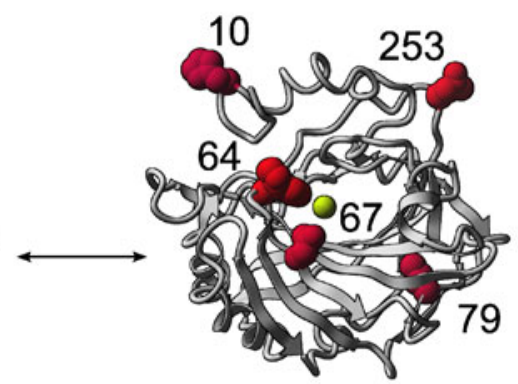

Unfolded

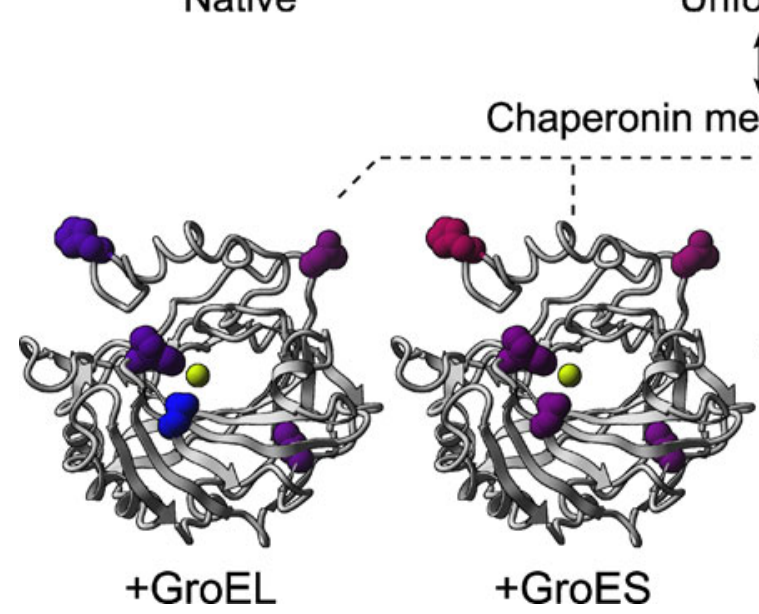

+ GroEL

+ GroES
Fig. 3 Local conformational interactions by chaperonin components with AEDANS-labeled HCAII variants during refolding. Spectra were recorded at equilibrium for native $(0.3 \mathrm{M} \mathrm{GuHCl})$ and for unfolded protein $(3.3 \mathrm{M} \mathrm{GuHCl})$ or within $60 \mathrm{~s}$ after initiation of refolding by manual mixing following direct excitation of AEDANS at $350 \mathrm{~nm}$. For experimental reasons, complete fluorescence spectra could only be recorded approximately $60 \mathrm{~s}$ after initiating refolding following manual mixing (20 s dead time). Reassuringly, ANS fluorescence from stoppedflow measurements still showed a considerable difference at this time point (Fig. 2). a The polarity, as reflected by the wavelength of the

formed GroEL/ES/HCAII complex (Fig. 3b, c). Apparently the interaction with GroEL and GroES has distinct effects on

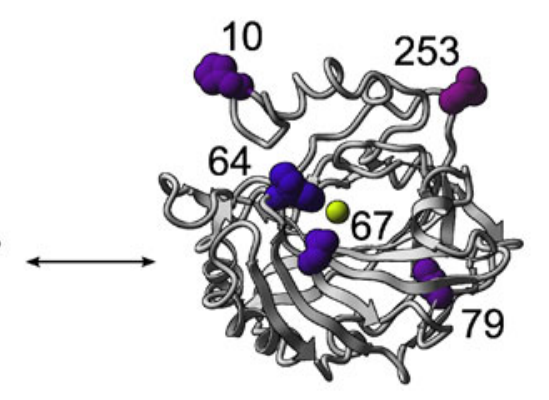

Spontaneous refolding

$\downarrow$
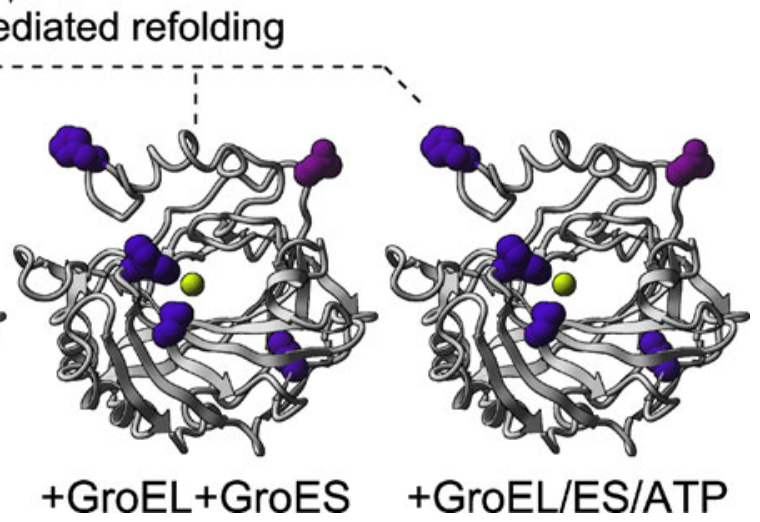

emission maximum, is illustrated in terms of the corresponding dielectric constant $\left(\varepsilon_{\mathrm{r}}\right)$ in a linear range of a blue-red color scale as determined by Stokes shifts in reference solvent experiments (see "Materials and methods"). b Distribution of AEDANS emission maxima during refolding of the single-cysteine HCAII variants at different GroE chaperon conditions. Colors for the various AEDANS-labeled sites: black position 10 , green position 64 , red position 67 , blue position 79 , and open position 253. $\mathrm{c}$ The AEDANS-labeled positions are highlighted, and the spectral shifts obtained during the various folding conditions are visualized using the blue-red $\varepsilon_{\mathrm{r}}$ color scale shown in a

the substrate, and subsequent formation of a GroEL/ES/ HCAII complex leads to a substantial remodeling of the 
substrate and/or altered interactions with both the chaperonin components.

Effects on substrate protein compactness by GroEL, GroES, and GroEL + GroES We have earlier shown that measurement of Trp-AEDANS FRET can be used to assess the compactness of HCAII at equilibrium during various stages of unfolding or during interaction with GroEL [5]. Therefore, FRET was calculated from fluorescence measurements, which were recorded during refolding of the five AEDANS-labeled HCAII variants, in order to obtain information on the structural modulation of the client protein by each individual chaperonin component and in the GroEL/ES/HCAII and GroEL/ES/ATP/HCAII complexes. The time span between $50 \mathrm{~ms}$ and $3 \mathrm{~h}$ of folding was covered by a combination of measurements after stopped-flow mixing and after manual mixing. The FRET values from all these conditions are compiled in Fig. 4 and Supplementary: Fig. S1. The FRET values are normalized so that a value of one indicates a fully folded native protein. In the following description of the results, we have interpreted a change in FRET value to indicate a change in compactness, i.e., an increase of the FRET value indicates a compaction of the protein and a decrease indicates a swelling/ expansion of the protein. In Fig. 4a, kinetic traces are shown from stopped-flow FRET experiments of the early stages of refolding $(50 \mathrm{~ms}-1 \mathrm{~s})$ for a representative AEDANS-labeled variant in the presence and absence of the chaperonin components. The magnitudes of the FRET values (at $50 \mathrm{~ms}$ ) are intermediate between what is observed for the unfolded and native states and essentially constant throughout the 1-s time frame. Thus, it is evident that spontaneous compaction of the protein (i.e., hydrophobic collapse) or formation of the different substrate complexes with the chaperonin components occurs well before the first data point $\left(50 \mathrm{~ms}, 2{ }^{\circ} \mathrm{C}\right)$.

Interactions with GroEL During GroEL-mediated folding, the HCAII substrate is less compact than during spontaneous refolding in the time span between $50 \mathrm{~ms}$ to $60 \mathrm{~s}$ (Fig. 4b, c). All mutants show this pattern, which demonstrates that interactions with GroEL alone essentially lead to an expansion of the entire HCAII molecule and that this expansion persists for a long time after the initial binding step. After completion of the refolding process $(3 \mathrm{~h})$, the situation is reversed. Here refolding in the presence of GroEL results in a compact native protein with a higher yield than after spontaneous refolding showing the GroEL-mediated chaperone effect (Fig. 4d).

Interactions with GroES Refolding in the presence of GroES initially ( $50 \mathrm{~ms}-60 \mathrm{~s}$ ) results in formation of a GroES/HCAII complex in which the environment of the central core positions (positions 67,79) is expanded compared to that in spontaneous refolding and the environment around the $\mathrm{C}$-terminus (position 253 ) is compacted (Fig. 4b, c). Thus, it is clear that GroES alone can modulate the substrate during refolding. However, after $3 \mathrm{~h}$ of refolding, in the presence or absence of GroES, the compactness of the HCAII molecules is very similar, indicating that GroES, despite initial binding and remodeling of the substrate, is not sufficient for supporting the complete folding process (Fig. 4d).

Interactions in the simultaneous presence of GroES and GroEL The observation that the client protein can interact with the chaperonin components individually provokes a new question: Which interactions are formed with the substrate when GroES and GroEL are both present? A comparison of the results obtained from measurements at $50 \mathrm{~ms}$ and $60 \mathrm{~s}$ on the labeled HCAII variants in the presence of GroEL + GroES with the corresponding measurements in the presence of only GroEL or GroES shows that the effects on the substrate compactness at various positions differ between these conditions (Fig. 4b, c). Thus, in the absence of ATP but in the presence of both the chaperonin components, HCAII forms a ternary GroEL/ES/ HCAII complex. Apparently, the interactions of HCAII with both GroEL and GroES are a necessary and sufficient requirement for formation of the ternary complex. A detailed analysis of the compactness at the labeled positions revealed distinct effects from interactions in the ternary complex. For example, at $50 \mathrm{~ms}$ the effects on compactness at positions 64 and 67 are very similar to the effects from GroEL alone, while the effect at position 253 is intermediate between the individual effects from GroES and GroEL. At 60 s, the effects at positions 10, 67, and 79 differ strongly from the effects of both GroEL and GroES, and in particular, one may notice that the interactions in the ternary complex lead to a strikingly expanded structure around core positions 67 and 79. Thus, an analysis of interactions with both GroEL and GroES in the ternary GroEL/ES/HCAII complex reveals that some new interactions with the substrate are formed upon formation of the ternary complex and that some of the substrate interactions that were observed with the individual chaperonin components are maintained.

To confirm that the effects caused by GroES and GroEL are due to specific binding, we measured FRET for the 253 variant, which show opposite effects by GroEL and GroES, during the refolding reaction in the presence of $10 \mu \mathrm{M}$ bovine serum albumin (Supplementary: Fig. S2). No BSA-dependent effects on compactness were detected, supporting the conclusion that the effects observed for the respective chaperonin components were not caused by non-specific interactions. Of note, the time courses for spontaneous and GroES-mediated reactivation coincide, demonstrating that GroES acting alone does not affect the final outcome of the refolding yield or the kinetics on this timescale (Supplementary: Fig. S3). Of significance, however, is the transient effect of GroES on compactness in the presence of GroEL even in the absence of ATP. Therefore, the role of GroES may be to direct the substrate toward more efficient binding in the ternary GroEL/ES/substrate complex. 
a
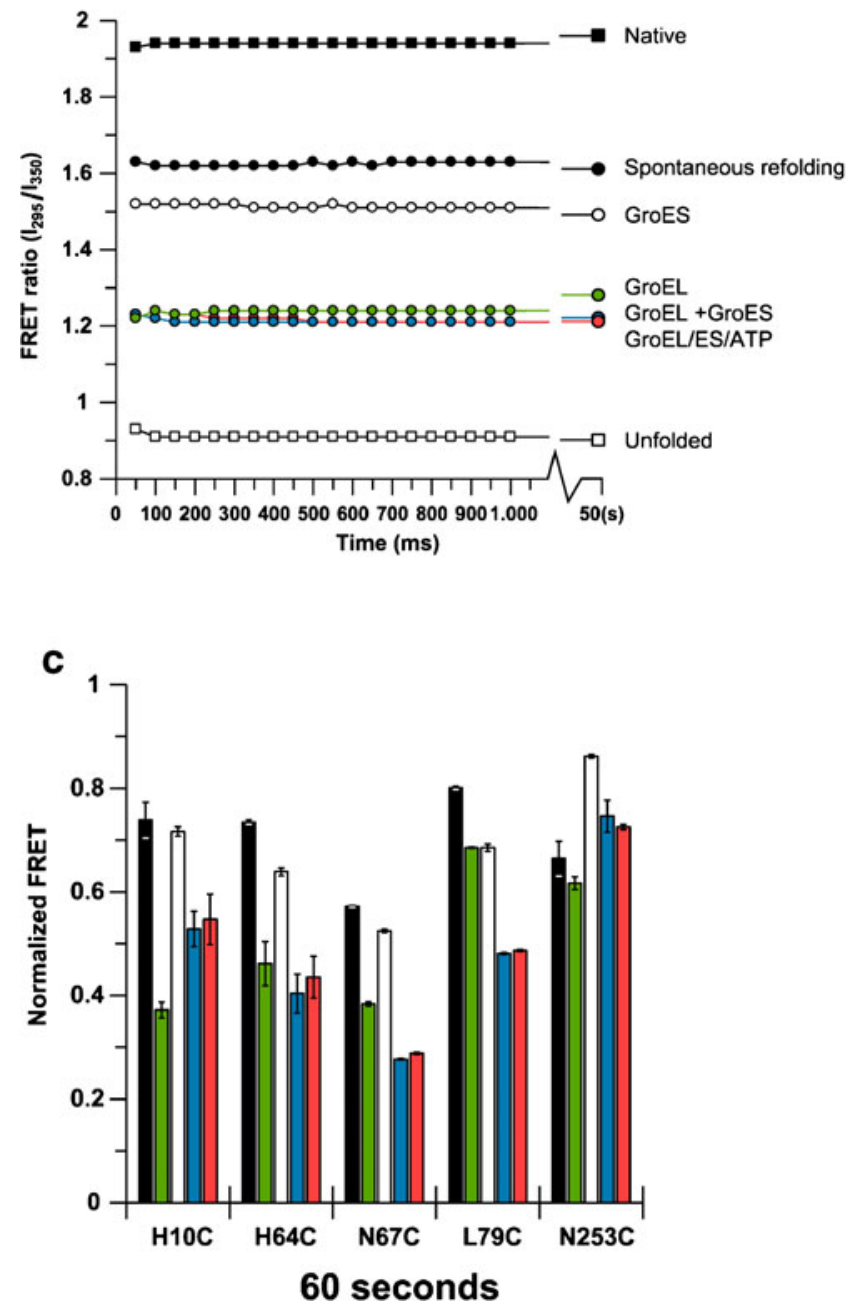

Fig. 4 Kinetic Trp-AEDANS FRET measurements of AEDANS-labeled HCAII. a A stopped-flow kinetic trace of the Trp-AEDANS FRET from the refolding of AEDANS-N67C (each line represents a calculated ratio from an average of seven runs for each excitation wavelength): black squares native protein (at $0.3 \mathrm{M} \mathrm{GuHCl}$ ), open squares unfolded protein (at $3.3 \mathrm{M} \mathrm{GuHCl}$ ), black circles spontaneous refolding, green circles with GroEL alone, open circles with GroES alone, blue circles with GroEL+ GroES, and red circles with GroEL/ES/ATP. b Fraction of folded HCAII mutants from stopped-flow measurements at $50 \mathrm{~ms}$ in the presence and absence of the chaperonin components with normalization of the calculated FRET done as explained in Supplementary: Fig.S1b: black

Interactions in the complete GroES/EL/ATP/substrate complex A comparison between the results obtained on the ternary GroES/EL/HCAII complex and on the GroES/EL/ ATP/HCAII complex shows a remarkably close similarity for all substrate variants and at all time points (Fig. 4b-d). It seems as if the presence of ATP does not enhance the efficiency of the GroEL/ES chaperonin on the HCAII substrate in contrast to what was found for MreB (Fig. 1c). The possibility that the intricate cooperation of GroES and GroEL in substrate

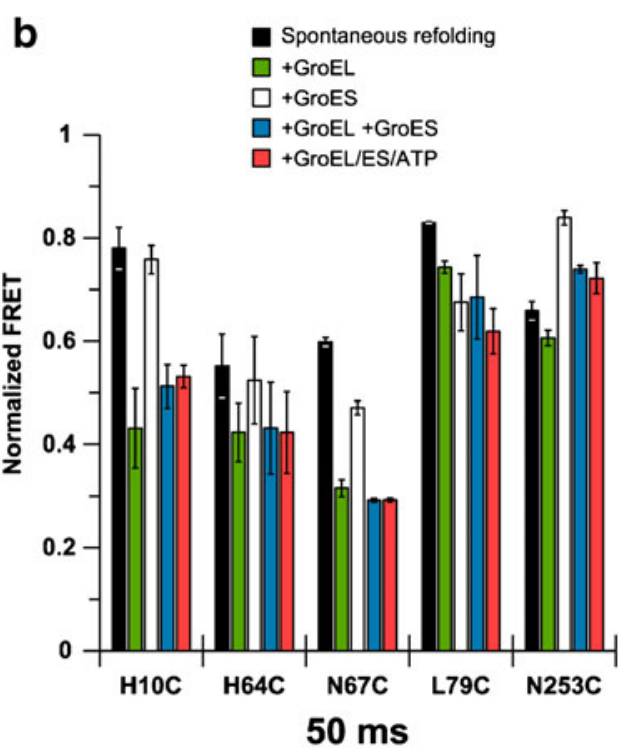

d

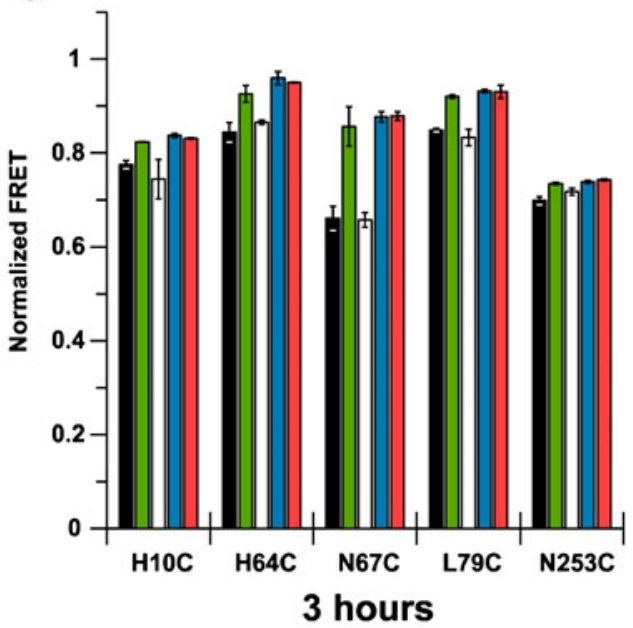

spontaneous refolding, green with GroEL alone, white with GroES alone, blue with GroEL+GroES, and red with GroEL/ES/ATP. c Fraction of folded AEDANS-labeled HCAII mutants after $60 \mathrm{~s}$ refolding time by manual mixing. The degree of refolding was determined from steadystate fluorescence spectra recorded in the presence and absence of the chaperonin components with normalization of the calculated FRET done as in $\mathbf{b}$ : The same color code as in $\mathbf{b}$ is used. $\mathbf{d}$ Fraction of folded AEDANS-labeled HCAII mutants after $3 \mathrm{~h}$ refolding time. The degree of refolding was determined from steady-state fluorescence spectra in the presence and absence of the chaperonin components with normalization of the calculated FRET done as in $\mathbf{b}$. The same color code as in $\mathbf{b}$ is used

binding and in the subsequent folding may be operative also with other substrates is an intriguing question!

\section{Discussion}

There is a common notion that the GroEL/ES chaperonin system entails cycles of strictly interdependent sequential steps or phases: GroEL capturing a substrate protein, ATP 
binding to form a GroEL/protein substrate complex followed by GroES binding as a lid on GroEL, and subsequent protein substrate release into the central cis-cavity to induce folding. The last step is ATP hydrolysis followed by release of the GroES lid and the folded protein substrate [48]. While this may be the dominant mechanism, we herein report on dynamic remodeling (expansion and compression) of a protein substrate by both GroEL and GroES individually and in combination during the folding process also in the absence of ATP.

Before a discussion of the reported results, it might be helpful to consider some earlier observations on the cellular concentrations of the chaperonin components under various cellular conditions. Neidhardt and VanBogelen have shown that under normal conditions $\left(37^{\circ} \mathrm{C}\right)$, the relative ratio of GroES versus GroEL is 1.9 and after heat shock 4.7 [49]. The normal concentration of GroEL is $2.6 \mu \mathrm{M}$ and that of GroES is $5.1 \mu \mathrm{M}$ [50], and upon heat induction (from 28 to $42{ }^{\circ} \mathrm{C}$ ), these numbers increase within $8 \mathrm{~min}$ to $20.5 \mu \mathrm{M}$ for GroEL and $96.9 \mu \mathrm{M}$ for GroES. Using a dissociation constant $K_{\mathrm{d}}=3 \mu \mathrm{M}$ for formation of a GroEL/ES complex in the absence of ATP as determined by Behlke et al. [51], it is notable that upon heat induction most of the GroEL is bound in a GroEL/ES complex leaving $>70 \mu \mathrm{M}$ free GroES in solution if bound on one side of GroEL and $>50 \mu \mathrm{M}$ if bound on both sides of GroEL. Note that the concentration of nonGroEL bound GroES after heat induction is virtually unchanged also in the presence of ATP. At ambient temperature applying the same reasoning, the concentration of free GroES is 2.5 or $0 \mu \mathrm{M}$, respectively. More recently, the Buchner and Winter groups reported that E. coli BM28 cells

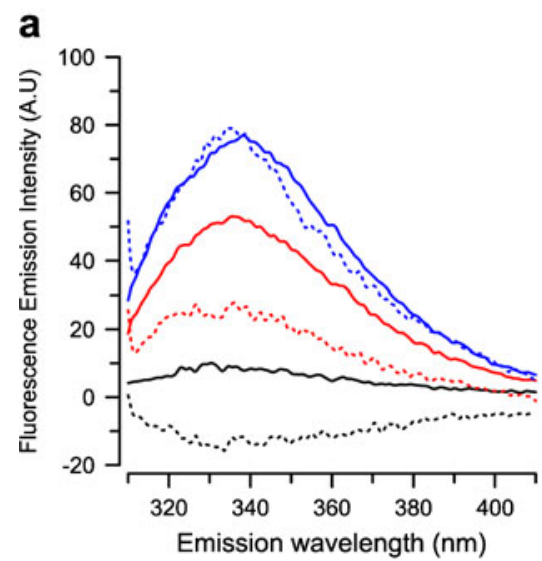

Fig. 5 Trp quenching of HCAII after manual mixing when refolded by stepwise titration at low concentrations in the presence of $1.0 \mu \mathrm{M}$ GroES98C-AEDANS. a Trp spectra represented by dotted lines in the presence and by solid lines in the absence of GroES-98C-AEDANS. Black $=$ $0.013 \mu \mathrm{M} ;$ red $=0.10 \mu \mathrm{M} ;$ blue $=0.17 \mu \mathrm{M}$ final concentration of HCAII. Also note the apparent blue-shift of the Trp spectrum at $0.17 \mu \mathrm{M}$ HCA II, which suggested that the fractional residual fluorescence originates from buried Trps. Experiments shown for unlabeled HCAII H64C/C206S mutant. b Stepwise titration of FRET efficiency during HCA II refolding contained $20 \mu \mathrm{g}$ GroEL and $19 \mu \mathrm{g}$ GroES upon heat shock [52]. Taking the 6-fold difference in molecular weight into account, this provides an 11.2-fold molar excess of GroES compared to GroEL providing putatively even higher numbers of free GroES than those discussed above. Taking our results on substrate protein binding to GroES into account, it would appear that GroES efficiently captures all or a subset of the cellular substrates during folding for subsequent interaction with GroEL and formation of a GroEL/ES/substrate complex or for degradation. Is there support for this notion? A semi-quantitative FRET measurement of the binding efficiency between GroES and folding HCAII shows an apparent dissociation constant of approximately $50 \mathrm{nM}$ at least for a subset of folding molecules (Fig. 5a, b), implicating the presence of very strong interacting conformers of the folding substrate with GroES. Interestingly, there are early reports implicating GroES-mediated folding of GroEL itself as revealed by an increased yield of GroEL folding [53]. Furthermore, it has been shown that Hsp10 mediates mitochondrial import of Hsp60 [54]. Intriguingly, it has recently been reported that chromosomally expressed bacterial mRNAs, in particular groESL mRNA, spatially remain near their site of transcription for their entire lifespan and that this localization restricts ribosomal mobility, implying that translation and thus protein synthesis of GroES and GroEL are spatially organized according to gene order [55]. Therefore, GroES will first be synthesized facilitating its interaction with the subsequently formed GroEL. It is hence intriguing to propose that GroEL is a substrate for GroES. The GroES activity by virtue of expression levels, most notably during stress conditions, would

\section{b}

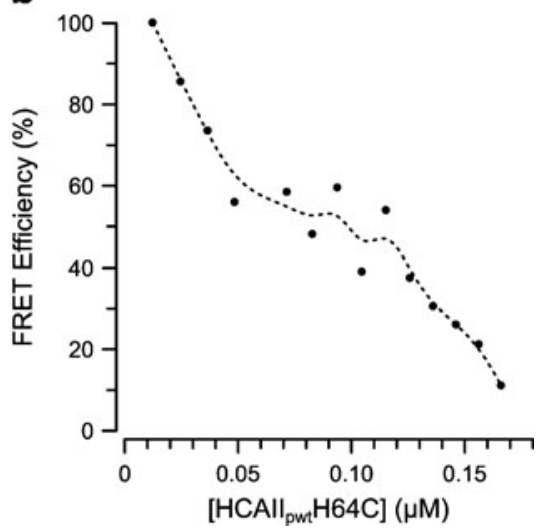

into a 1.0- $\mu \mathrm{M}$ GroES-98C-AEDANS buffer solution (data such as those in a). The FRET efficiency (in percent) was determined by the donor quenching $(E=1-(\mathrm{FDA} / \mathrm{FD}) \times 100)$. FRET is efficient at low concentrations of HCA II showing that GroES has very high affinity at least for a sub-population of HCA II molecules during folding, indicating a $K_{\mathrm{d}}$ of approximately $50 \mathrm{nM}$, where a plateau becomes apparent (between 50 and $120 \mathrm{nM}$ ). At higher concentrations, the background fluorescence of Trp (from refolded molecules) becomes dominant and is likely the reason why the observed FRET efficiency drops off 
a

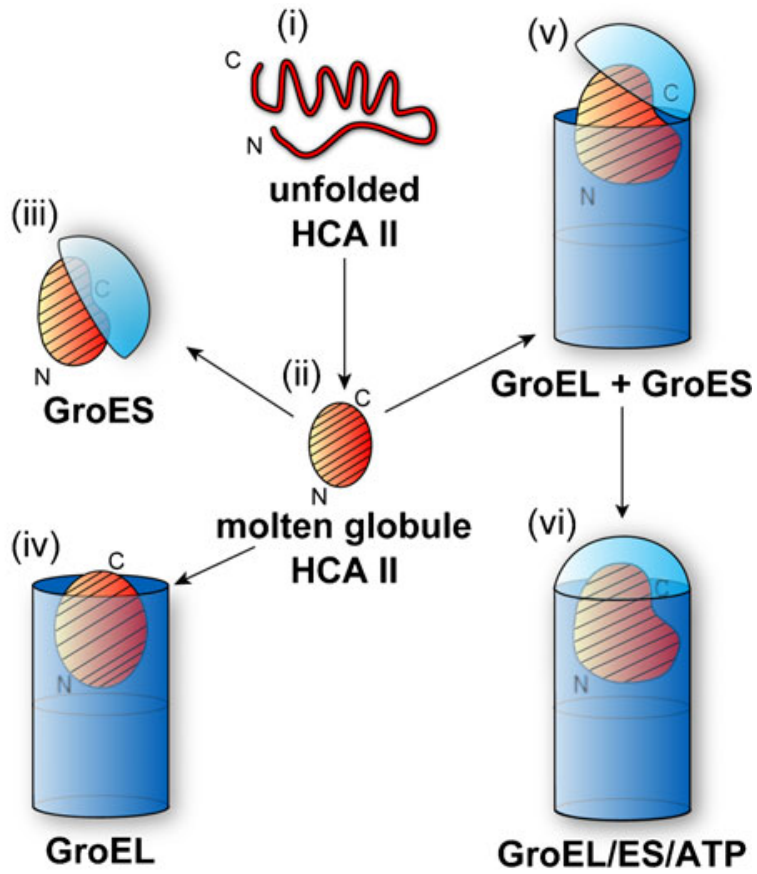

b
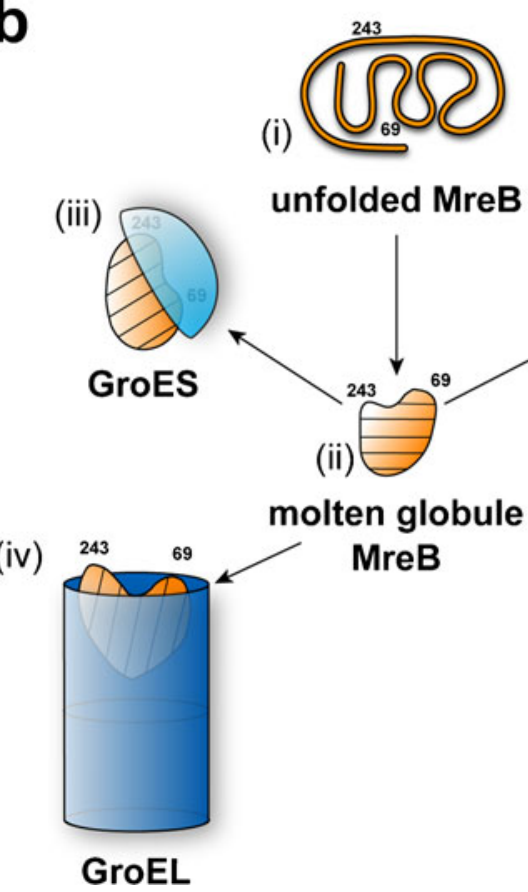

unfolded MreB

(v)

(ii)

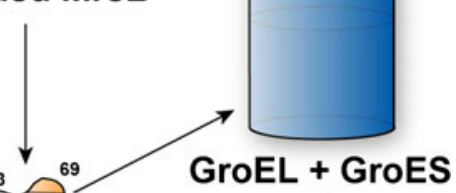

MreB

(vi)

(vi)

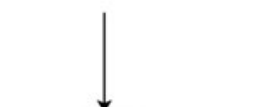

be further enhanced on a polysome. The large excess of GroES in vivo during temperature stress reported by several groups discussed above implicates an until now unknown important role in cell viability.

It was recently suggested that the unfolded protein in the cis-cavity partly protrudes through a narrow space near the GroEL/ES interface [56] and some examples exist in the
4 Fig. 6 Schematic cartoon describing global shapes of the protein substrates during refolding in the presence and absence of chaperonins. a HCAII during refolding in the presence and absence of chaperonins in the time frame of $50 \mathrm{~ms}-60 \mathrm{~s}$. Top center $(i)$ : Unfolded HCAII is represented by an ensemble of distorted structures with some residual structure of its central $\beta$-strands condensed by the hydrophobic core [61]. Center (ii): Directly after refolding, the refolding HCAII undergoes a hydrophobic collapse (spontaneous refolding) into a molten globule state. $N$ and $C$ mark the location of the protein termini. Top left (iii): The GroES interaction alone renders a conformational change toward a more expanded core as well as a compression of the $\mathrm{C}$-terminal region. Bottom left (iv): The GroEL interaction alone renders a conformational change toward a highly expanded core. The orientation of HCAII in the GroEL cavity is likely stochastic but is herein shown in relation to GroEL+ GroES (see below). Polarity data supports extensive burial of position 67. Top right $(v)$ : The concerted GroEL + GroES interaction enforces a conformational change toward a highly expanded core as well as a compression of the $\mathrm{C}$-terminal region. The concerted action of GroEL and GroES suggests a likely orientation of HCAII where the N-terminal subdomain is more buried than the C-terminal region which appears accessible for GroES and should hence point outward from the GroEL cavity. Bottom right (vi): The complete chaperonin system GroEL/ES/ ATP renders a very similar morphological change as in the absence of ATP, but most likely involves encapsulation of HCAII into the cis-cavity. b MreB during refolding in the presence and absence of chaperonins. Top center $(i)$ : Unfolded MreB is represented by an ensemble of distorted structures with the indicated fluorescein labeled positions 69 and 243. Center (ii): Directly after refolding the refolding MreB undergoes a hydrophobic collapse (spontaneous refolding) into a partially folded molten globule state. Top left (iii): The GroES interaction alone renders a conformational change toward an expanded molecule. Bottom left (iv): The GroEL interaction alone renders a conformational change toward an expanded molecule. Top right (v): The concerted GroEL+GroES interaction renders a conformational change toward an expanded molecule. Bottom right (vi): Addition of ATP will afford the complete chaperonin system GroEL/ES/ATP and facilitates a compression of MreB during encapsulation within the $c i s$-cavity

literature on the conformational consequences for the substrate during the GroEL cycle [57, 58]. Importantly, it has never previously been reported of independent binding of GroES to a folding substrate protein. In this work, several experimental evidences are presented that unequivocally demonstrate that the investigated protein substrates are captured by GroES alone. For the E. coli protein MreB, GroES binding was shown by elevated fluorescence anisotropy and decreased HOMO-FRET. For the folding model protein HCAII, this effect was shown by red-shifted AEDANS fluorescence, suppressed ANS-binding, altered intramolecular FRET efficiency, and intermolecular HCAII-GroES FRET. Herein, the folding of HCAII was thoroughly investigated in the presence and absence of chaperonins using stopped-flow and manual mixing experiments. The results led us to conclude that GroEL mediates an expansion of the client protein, i.e., acts as an unfoldase, at an early stage in the folding process relative to spontaneous folding which translates later on to elevated refolding yields. This unfoldase activity has been established by many groups over the years both at equilibrium, e.g., [4-6, 
$59,60]$ and recently during folding [8]. Furthermore, we showed that GroES influences the remodeling of two client proteins when acting in concert with GroEL. Thus, GroES acts as a modulator of GroEL function, although GroES alone despite interacting with the molten globule does not have complete chaperone activity per se. Despite differences in experimental approaches and protein substrates, the conformational data on MreB and HCAII during these steps are almost identical as schematically summarized in Fig. 6a, b. Nevertheless, differences were found. The C-terminal part of HCAII was compressed by GroES, and in the case of MreB, we observed an ATP-mediated compaction when ATP was added to the ternary complex. In summary, the concerted interactions by GroES and GroEL appear to enhance an expansion of the initially collapsed molten globule state. Thus, this will provide the protein substrate with a new chance to fold correctly and avoid misfolding side steps off the productive folding pathway [61]. Our results necessitate a partial revision of the mechanism of function of the GroE chaperonin system. In particular, we suggest a more dynamic role to GroES which is likely more important than merely as a cochaperone to GroEL. How is it possible that these effects by
GroES alone have evaded the chaperonin community for over two decades? It was of course possible that our findings by mere serendipity were limited to the two client proteins both which are approximately $30 \mathrm{kDa}$ despite their difference in function, fold, and sequence. With this in mind, we performed a study of four additional proteins with a range of molecular weights between 14 and $150 \mathrm{kDa}$ : lysozyme, BSA, DNAse I, and $\gamma$-globulin. The proteins were refolded from $5 \mathrm{M} \mathrm{GuHCl}$ in the presence or absence of 1,5-IAEDANS-labeled GroES, GroES-98C-AEDANS. For all four proteins, the Trp fluorescence after $1 \mathrm{~min}$ of refolding was lower in the presence of GroES-98C-AEDANS compared to in the absence (Fig. 7a-d). The most striking quenching was observed for lysozyme (Fig. 7a) and BSA (Fig. 7c) supporting that GroES is binding to at least these two proteins during folding. It is hence very tempting to draw the conclusion that GroES is actively participating during folding for many clients (in our study at least four out of six very different proteins). The reason for why this function has not been reported in the literature is likely due to the transient nature of the interaction and the imperative role for GroEL in facilitating elevated refolding yields in vitro.
Fig. 7 Trp quenching of four proteins when refolded at $0.5 \mu \mathrm{M}$ concentrations in the presence of $1.0 \mu \mathrm{M}$ GroES-98C-AEDANS. Herein we compare the spontaneous refolding (solid red line) with the refolding in the presence of GroES-98CAEDANS (dotted black line). There is substantial quenching of a lysozyme and $\mathbf{c}$ BSA, some for b DNAse I, and very little for $\mathbf{d}$ $\gamma$-globulin
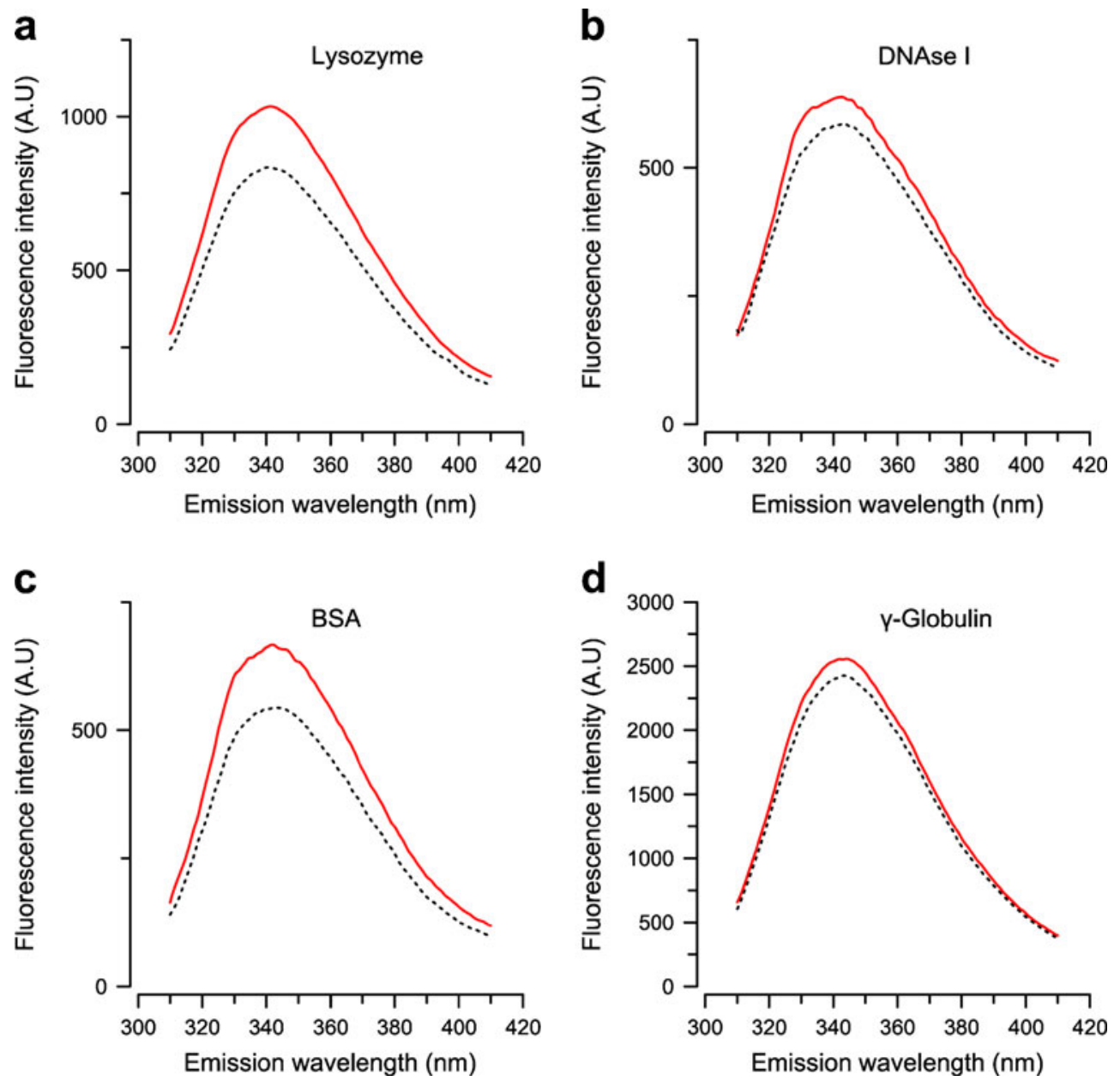
Acknowledgments We thank Dr. George H. Lorimer for the kind gift of the GroES-C98 plasmid and helpful suggestions on making direct GroES-HCAII FRET measurements. We thank Åsa Källstrand Thor for artistic work on the cartoons in Fig. 6. This work was supported by the Swedish Research Council (U.C., P.H., B.H.J.), the European Community FP6 through a Marie Curie EST fellowship under contract number MEST-CT-2004-504272 (SBM), Knut och Alice Wallenberg Foundation (P.H., U.C., B.H.J.), and Stiftelsen Marcus och Amalia Wallenbergs Minnesfond (U.C.). P.H. was supported as a Swedish Royal Academy of Science Research Fellow sponsored by a grant from the Knut and Alice Wallenberg Foundation.

Open Access This article is distributed under the terms of the Creative Commons Attribution License which permits any use, distribution, and reproduction in any medium, provided the original author(s) and the source are credited.

\section{References}

1. Ewalt KL, Hendrick JP, Houry WA, Hartl FU (1997) In vivo observation of polypeptide flux through the bacterial chaperonin system. Cell 90:491-500

2. Kerner MJ, Naylor DJ, Ishihama Y, Maier T, Chang HC, Stines AP, Georgopoulos C, Frishman D, Hayer-Hartl M, Mann M, Hartl FU (2005) Proteome-wide analysis of chaperonin-dependent protein folding in Escherichia coli. Cell 122:209-220

3. Persson M, Aronsson G, Bergenhem N, Freskgård P-O, Jonsson B-H, Surin BP, Spangfort MD, Carlsson U (1995) GroEL/ES-mediated refolding of human carbonic anhydrase II: role of $\mathrm{N}$-terminal helices as recognition motifs for GroEL. Biochim Biophys Acta 1247:195-200

4. Persson M, Hammarström P, Lindgren M, Jonsson B-H, Svensson M, Carlsson U (1999) EPR mapping of interactions between spinlabeled variants of human carbonic anhydrase II and GroEL: evidence for increased flexibility of the hydrophobic core by the interaction. Biochemistry 38:432-441

5. Hammarström P, Persson M, Carlsson U (2001) Protein compactness measured by fluorescence resonance energy transfer. Human carbonic anhydrase is considerably expanded by the interaction of GroEL. J Biol Chem 276:21765-21775

6. Owenius R, Jarl A, Jonsson B-H, Carlsson U, Hammarström P (2010) GroEL-induced topological dislocation of a substrate protein $\beta$-sheet core: a solution EPR spin-spin distance study. J Chem Biol 3: 127-139

7. Villebeck L, Moparthi SB, Lindgren M, Hammarström P, Jonssson B-H (2007) Biochemistry 46:1269-12647

8. Priya S, Sharma SK, Sood V, Mattoo RU, Finka A, Azem A, De Los RP, Goloubinoff P (2013) GroEL and CCT are catalytic unfoldases mediating out-of-cage polypeptide refolding without ATP. Proc Natl Acad Sci U S A 110:7199-7204

9. Badcoe IG, Smith CJ, Wood S, Halsall DJ, Holbrook JJ, Lund P, Clarke AR (1991) Binding of a chaperonin to the folding intermediates of lactate dehydrogenase. Biochemistry 30:9195-9200

10. Brown KL, Wood S, Buttner MJ (1992) Isolation and characterization of the major vegetative RNA polymerase of Streptomyces coelicolor A3(2); renaturation of a sigma subunit using GroEL. Mol Microbiol 6:1133-1139

11. Hansen JE, Gafni A (1993) Thermal switching between enhanced and arrested reactivation of bacterial glucose-6-phosphate dehydrogenase assisted by GroEL in the absence of ATP. J Biol Chem 268: 21632-21636

12. Gray TE, Fersht AR (1993) Refolding of barnase in the presence of GroE. J Mol Biol 232:1197-1207
13. Persson M, Carlsson U, Bergenhem NC (1996) GroEL reversibly binds to, and causes rapid inactivation of, human carbonic anhydrase II at high temperatures. Biochim Biophys Acta 1298:191-198

14. Krauss O, Gore MG (1996) Refolding and reassociation of glycerol dehydrogenase from Bacillus stearothermophilus in the absence and presence of GroEL. Eur J Biochem 241:538-545

15. Sparrer H, Lilie H, Buchner J (1996) Dynamics of the GroEL-protein complex: effects of nucleotides and folding mutants. J Mol Biol 258: $74-87$

16. Viitanen PV, Donaldson GK, Lorimer GH, Lubben TH, Gatenby AA (1991) Complex interactions between the chaperonin 60 molecular chaperone and dihydrofolate reductase. Biochemistry 30:9716-9723

17. Fisher MT (1992) Promotion of the in vitro renaturation of dodecameric glutamine synthetase from Escherichia coli in the presence of GroEL (chaperonin-60) and ATP. Biochemistry 31: 3955-3963

18. Goloubinoff P, Gatenby AA, Lorimer GH (1989) GroE heat-shock proteins promote assembly of foreign prokaryotic ribulose bisphosphate carboxylase oligomers in Escherichia coli. Nature 337:44-47

19. Goloubinoff P, Christeller JT, Gatenby AA, Lorimer GH (1989) Reconstitution of active dimeric ribulose bisphosphate carboxylase from an unfolded state depends on two chaperonin proteins and $\mathrm{Mg}$ ATP. Nature 342:884-889

20. Todd MJ, Lorimer GH, Thirumalai D (1996) Chaperonin-facilitated protein folding: optimization of rate and yield by an iterative annealing mechanism. Proc Natl Acad Sci U S A 93:4030-4035

21. Shtilerman M, Lorimer GH, Englander SW (1999) Chaperonin function: folding by forced unfolding. Science $284: 822-825$

22. Elad N, Farr GW, Clare DK, Orlova EV, Horwich AL, Saibil HR (2007) Topologies of a substrate protein bound to the chaperonin GroEL. Mol Cell 26:415-426

23. Chen DH, Madan D, Weaver J, Lin Z, Schröder GF, Chiu W, Rye HS (2013) Visualizing GroEL/ES in the act of encapsulating a folding protein. Cell 153:1354-1365

24. Laminet AA, Ziegelhoffer T, Georgopoulos C, Pluckthun A (1990) The Escherichia coli heat shock proteins GroEL and GroES modulate the folding of the beta-lactamase precursor. EMBO J 9:2315-2319

25. Martin J, Langer T, Boteva R, Schramel A, Horwich AL, Hartl FU (1991) Chaperonin-mediated protein folding at the surface of groEL through a 'molten globule'-like intermediate. Nature 352:36-42

26. Frieden C, Clark AC (1997) Protein folding: how the mechanism of GroEL action is defined by kinetics. Proc Natl Acad Sci U S A 94: $5535-5538$

27. Aoki K, Motojima F, Taguchi H, Yomo T, Yoshida M (2000) GroEL binds artificial proteins with random sequences. J Biol Chem 275: $13755-13758$

28. Zhang X, Beuron F, Freemon PS (2002) Machinery of protein folding and unfolding. Curr Opin Struct Biol 12:231-238

29. Lin Z, Rye HS (2006) GroEL-mediated protein folding: making the impossible, possible. Crit Rev Biochem Mol Biol 41:211-239

30. Svensson M, Jonasson P, Freskgård PO, Jonsson BH, Lindgren M, Mårtensson LG, Gentile M, Borén K, Carlsson U (1995) Mapping the folding intermediate of human carbonic anhydrase II. Probing substructure by chemical reactivity and spin and fluorescence labeling of engineered cysteine residues. Biochemistry 34:8606-8620

31. van den Ent F, Löwe J (2006) RF cloning: a restriction-free method for inserting target genes into plasmids. J Biochem Biophys Methods 67:67-74

32. Riddles PW, Blakeley RL, Zerner B (1983) Reassessment of Ellman's reagent. Methods Enzymol 91:49-60

33. Freskgård PO, Carlsson U, Mårtensson LG, Jonsson BH (1991) Folding around the C-terminus of human carbonic anhydrase II. Kinetic characterization by use of a chemically reactive SH-group introduced by protein engineering. FEBS Lett 289:117-122 
34. Mårtensson LG, Karlsson M, Carlsson U (2002) Dramatic stabilization of the native state of human carbonic anhydrase II by an engineered disulfide bond. Biochemistry 41:15867-15875

35. Mårtensson LG, Jonasson $\mathrm{P}$, Freskgård $\mathrm{PO}$, Svensson $\mathrm{M}$, Carlsson U, Jonsson BH (1995) Contribution of individual tryptophan residues to the fluorescence spectrum of native and denatured forms of human carbonic anhydrase II. Biochemistry 34:1011-1021

36. Mårtensson LG, Jonsson BH, Andersson M, Kihlgren A, Bergenhem N, Carlsson U (1992) Role of an evolutionarily invariant serine for the stability of human carbonic anhydrase II. Biochim Biophys Acta 1118:179-186

37. Villebeck L, Persson M, Luan SL, Hammarström P, Lindgren M, Jonsson BH (2007) Conformational rearrangements of tail-less complex polypeptide 1 (TCP-1) ring complex (TRiC)-bound actin. Biochemistry 46:5083-5093

38. Wohlfarth C (2008) Dielectric constant of the mixture (1) water; (2) ethanol. In: Lechner MD (ed) Springer materials-The LandoltBörnstein Database. Springer, Berlin. doi:10.1007/978-3-54075506-7_328, http://www.springermaterials.com

39. Håkansson K, Carlsson M, Svensson LA, Liljas A (1992) Structure of native and apo carbonic anhydrase II and structure of some of its anion-ligand complexes. J Mol Biol 227:1192-1204

40. Moparthi SB (2007) Dynamic remodeling of substrate protein by GroES during folding - additive transient stretching in concert with GroEL. 63rd Harden Conference - protein folding and assembly in vitro and in vivo. 18-23 August 2007, University of Cumbria, Ambleside, UK

41. Hammarström P, Persson M, Owenius R, Lindgren M, Carlsson U (2000) Protein substrate binding induces conformational changes in the chaperonin GroEL. A suggested mechanism for unfoldase activity. J Biol Chem 275:22832-22838

42. Albert A, Yunta C, Arranz R, Peña A, Salido E, Valpuesta JM, Martín-Benito J (2010) Structure of GroEL in complex with an early folding intermediate of alanine glyoxylate aminotransferase. J Biol Chem 285:6371-6376

43. Krishnamurthy VM, Kaufman GK, Urbach AR, Gitlin I, Gudiksen KL, Weibel DB, Whitesides GM (2008) Carbonic anhydrase as a model for biophysical and physical-organic studies of proteins and protein-ligand binding. Chem Rev 108:946-1051

44. Semisotnov GV, Rodionova NA, Kutyshenko VP, Ebert B, Blanck J, Ptitsyn OB (1987) Sequential mechanism of refolding of carbonic anhydrase B. FEBS Lett 222:9-13

45. Semisotnov GV, Rodionova NA, Razgukaev OI, Uversky VN, Gripas AF, Gilmanshin RI (1991) Study of the "molten globule" intermediate state in protein folding by a hydrophobic fluorescent probe. Biopolymers 31:119-128

46. Karlsson M, Mårtensson LG, Olofsson P, Carlsson U (2004) Circumnavigating misfolding traps in the energy landscape through protein engineering: suppression of molten globule and aggregation in carbonic anhydrase. Biochemistry 43:6803-6807
47. Hammarström P, Owenius R, Mårtensson LG, Carlsson U, Lindgren M (2001) High-resolution probing of local conformational changes in proteins by the use of multiple labeling: unfolding and self-assembly of human carbonic anhydrase II monitored by spin, fluorescent, and chemical probes. Biophys J 80:2867-2885

48. Horwich AL, Fenton WA (2009) Chaperonin-mediated protein folding: using a central cavity to kinetically assist polypeptide chain folding. Q Rev Biophys 42:83-116

49. Neidhardt FC, VanBogelen RA (1987) Heat shock response. In: Neidhardt FC, Ingraham JL, Low KB, Magasanik B, Schaechter M, Umbarger HE (eds) Escherichia coli and Salmonella typhimurium: cellular and molecular biology. American Society for Microbiology, Washington, DC, pp 1334-1345

50. Lorimer GH (1996) A quantitative assessment of the role of the chaperonin proteins in protein folding in vivo. FASEB J 10:5-9

51. Behlke J, Ristau O, Schönfeld HJ (1997) Nucleotide-dependent complex formation between the Escherichia coli chaperonins GroEL and GroES studied under equilibrium conditions. Biochemistry 36:5149-5156

52. Rudolph B, Gebendorfer KM, Buchner J, Winter J (2010) Evolution of Escherichia coli for growth at high temperatures. J Biol Chem 285:19029-19034

53. Lissin NM, Venyaminov SY, Girshovich AS (1990) (Mg-ATP)-dependent self-assembly of molecular chaperone GroEL. Nature 348:339-342

54. Dubaquié Y, Looser R, Fünfschilling U, Jenö P, Rospert S (1998) Identification of in vivo substrates of the yeast mitochondrial chaperonins reveals overlapping but non-identical requirement for hsp60 and hsp10. EMBO J 17:5868-5876

55. Montero Llopis P, Jackson AF, Sliusarenko O, Surovtsev I, Heinritz J, Emonet T, Jacobs-Wagner C (2010) Spatial organization of the flow of genetic information in bacteria. Nature 466:77-82

56. Motojima F, Yoshida M (2010) Polypeptide in the chaperonin cage partly protrudes out and then folds inside or escapes outside. EMBO J 29:4008-4019

57. Lin Z, Rye HS (2004) Expansion and compression of a protein folding intermediate by GroEL. Mol Cell 16:23-34

58. Sharma S, Chakraborty K, Müller BK, Astola N, Tang YC, Lamb DC, Hayer-Hartl M, Hartl FU (2008) Monitoring protein conformation along the pathway of chaperonin-assisted folding. Cell 133:142-153

59. Zahn R, Spitzfaden C, Ottiger M, Wüthrich K, Plückthun A (1994) Destabilization of the complete protein secondary structure on binding to the chaperone GroEL. Nature 368:261-265

60. Zahn R, Perrett S, Stenberg G, Fersht AR (1996) Catalysis of amide proton exchange by the molecular chaperones GroEL and SecB. Science 271:642-645

61. Hammarström P, Carlsson U (2000) Is the unfolded state the Rosetta stone of the protein folding problem? Biochem Biophys Res Commun 276:393-398

62. van den Ent F, Amos LA, Löwe J (2001) Prokaryotic origin of the actin cytoskeleton. Nature 413:39-44 